\title{
Cerebral proliferative angiopathy
}

\author{
Hugo Leonardo Dória-Netto ', Antônio Marcos de Souza-Filho', \\ Ricardo Henrique Dória-Netto ${ }^{1}$, Rômulo Alberto Silva Marques ${ }^{1}$, \\ Denise Aparecida de Oliveira ${ }^{2}$, Feres Chaddad-Neto ${ }^{3}$, \\ Christiane Monteiro de Siqueira Campos ${ }^{4}$
}

Since Cushing and Dandy reported their cases about "monstrous" vascular malformations with high mortality index in 1928, neurosurgery has been dedicating efforts to study such complex pathology among the most important ones in the modern medicine $^{1}$. Cerebral vascular malformation is a relatively rare pathology and its prevalence is difficult to estimate since a few number of affected individuals remain symptomatic. The classification contemplates size, local and venous draining territory ${ }^{2}$. Those malformations are congenital results of an anomalous cerebral development. Physiopathology, angioarchitecture and natural history distinguish many types of malformations such as arteriovenous malformations (AVM), venous angiomas, cavernous malformations, dural arteriovenous fistulas and capillary telangiectasy ${ }^{1,2}$. The AVMs are dilated tortuous vessel masses characterized by direct connections between arteries and veins (shunts) without a capillary net interposition ${ }^{1}$. A peculiar type of large brain AVM with multiple non dominant arterial feeders to a lobar or hemispheric nidus, relatively small draining veins associated to intermingled normal brain and angiographic evidence of capillary angioectasia and angiogenetic activity determines an atypical entity named cerebral proliferative angiopathy $(\mathrm{CPA})^{3}$. Rare vascular pathologies associated with skin and other organ lesions designate neurocutaneous angiomatosis syndrome ${ }^{2}$. Sturge Weber syndrome (SWS) and Rendu-Osler-Weber syndrome (ROWS) are examples of $i t^{4,5}$.
We report a female with an atypical diffuse lobar cerebral proliferative angiopathy (CPA).

\section{CASE}

A 39-years-old white female was admitted to our department presenting a chronic left, pulsatil, highly intense hemicrania headache. Physical practice increased it. She presented recurrent episodes of left hemiparesis and hemihypoestesia stopping completely in one to three hours. Left hemiparestesia was permanent. She smokes chronically. Neither was hypertension related nor convulsions.

Computed tomography $(\mathrm{CT})$ revealed a high density image in the left frontal cortex with contrast enhancement suggesting an AVM nidus.

Magnetic resonance image (MRI) did not characterize a typical AVM nidus, but a diffuse network of densely enhancing vascular spaces with intermingled normal brain parenchyma among different vascular territories (watershed zones) on the left frontal lobe, lower and medium frontal gyri, pre-central gyrus and basial ganglia (Fig 1).

MRA (Fig 2) and angiography showed a spread angiopathy supplied by numerous arterial branches leading to late venous filling (Fig 3), without markedly dilated superficial or deep cerebral veins, draining to superior sagittal sinus and Rosenthal basal veins (Fig 4). There was a diffuse cortical-subcortical capillary angioectatic net spread to the whole left frontal lobe (Fig 4)
Hugo Leonardo Dória-Netto Instituto de Ciências Neurológicas (ICNE) Praça Amadeu Amaral $27 / 5^{\circ}$ andar 01327-010 São Paulo SP - Brasil E-mail: hl.doria@uol.com.br fereschaddad@hotmail.com

\section{ANGIOPATIA CEREBRAL PROLIFERATIVA}

Neurology and Neurosurgery Department of Hospital Municipal Dr. Mário Gatti, Campinas SP, Brasil: ${ }^{1}$ Resident of Neurosurgery of Hospital Municipal Dr. Mário Gatti, Campinas SP, Brasil; ${ }^{2}$ Resident of Neuropediatry of Hospital das Clínicas, Universidade de São Paulo, São Paulo SP, Brazil; ${ }^{3}$ Neurosurgeon of Instituto de Ciencias Neurologicas (ICNE), Hospital Beneficência Portuguesa, São Paulo SP, Brazil and Hospital Municipal Dr. Mario Gatti, Campinas SP, Brasil; ${ }^{4}$ Neuroradiologist of Med Imagem, Hospital Beneficência Portuguesa, São Paulo SP, Brazil. 


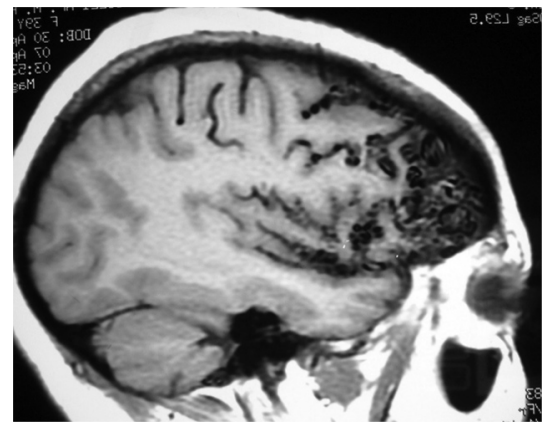

Fig 1. MRI, sagittal T1 exposing of multiple abnormal vessels within the sulci in the left frontal lobe.

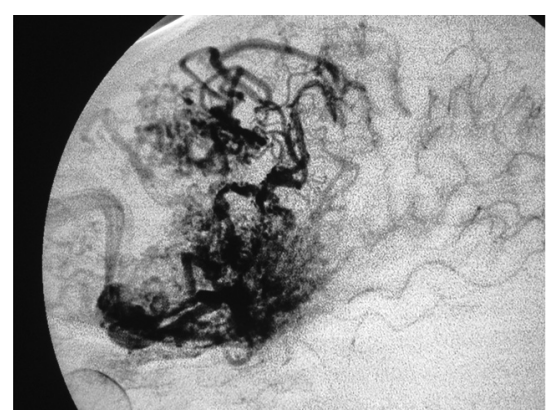

Fig 4. Angiographic late capillary phase in which there is still capillary angioectatic flow, confirming the delayed blood transit time and without markedly dilated draining veins.

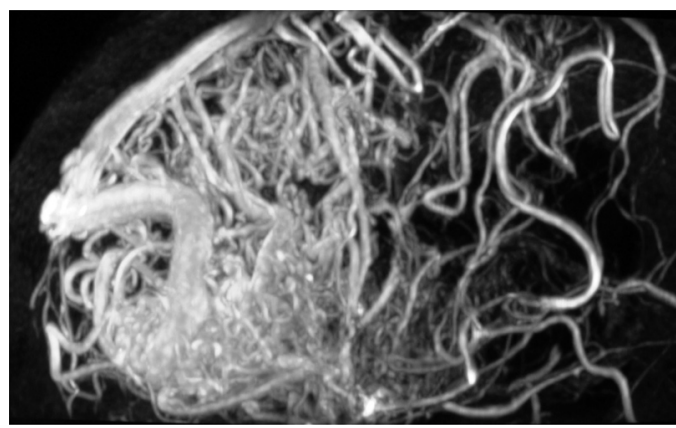

Fig 2. MRA Sagittal vision revealing cortical-subcortical densely enhanced network comprising the diffuse CPA. It is evident the hyperperfusion and angioectasy in left hemisphere.

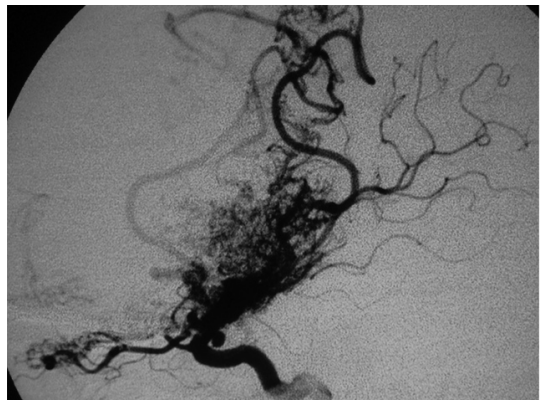

Fig 5. Angiographic evidence of ophthalmic arterial angiomatosis. Cerebral Aneurysms in ICA principal branch.

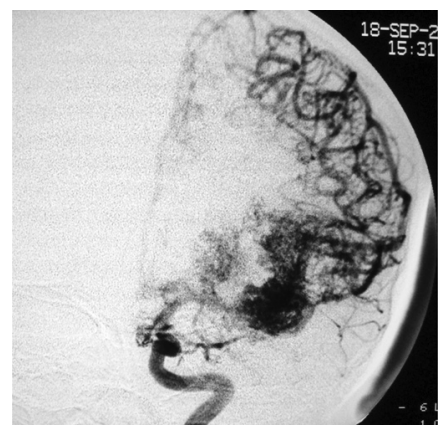

Fig 3. Irrigation arterial system composed of the MCA, Opht.A, ACA and lenticulostriate left branches. There is evidence of non dominant multiple arterial feeders filling the capillary angioectatic net.

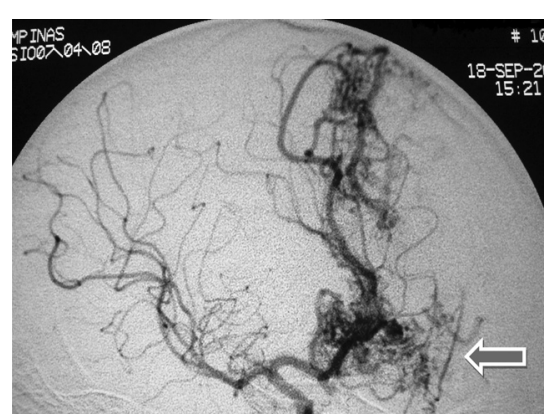

Fig 6. Angiographic frame of right ICA with relevant contribution to left CPA, characteristics of hemodynamic steal phenomenon. Evidence to Falcine artery contribution to CPA transdural supply testifying for the angiogenic proliferative nature of the disease. with intra orbital extension (Fig 5). Irrigation did not reveal a dominant arterial feeder, predominating over the left medial cerebral artery, left anterior choroid artery, left ophthalmic artery, anterior cerebral arteries and perforating branches to basal nuclei (Fig 3). Irrigation contribution by falcine anterior artery, from ophthalmic artery, and right anterior cerebral artery, is a typical pattern of hemodynamic steal phenomenon (Fig 6). Left internal carotid aneurysms are evident (Fig 5).

Symptomatic medical treatment failed. Patient's sixmonth outcome revealed permanent headache, dizziness, left parestesis and paroxysmal weakness without epileptic seizures.

\section{DISCUSSION}

Our patient suffers from an atypical angiopathy which differs from other AVM in their angiomorphology, histology, presumed mechanism, epidemiology, natural history and clinical presentation. It may therefore be classified as a distinct group of AVM.
Efforts made towards specific diagnosis include careful analysis of angiomatous syndromes.

Hereditary hemorrhagic telangiectasy, or Rendu-Osler-Weber syndrome, Sturge Weber syndrome, the Wyburn Mason syndrome and the angiomatosis of Divry Van Bogaert (ADB) are some vasculopathies that should be among differential diagnosis ${ }^{2,5,6}$.

Although these rare syndromes share some features with our patient, none of them entirely resembles.

Cerebral proliferative angiopathy appears as a presumed diagnosis for a peculiar type of large brain AVMs, predominating at $3.4 \%$ of them, $67 \%$ being females ${ }^{3}$.

Clinical aspects point to small chance of hemorrhage $12 \%, 45 \%$ of seizures, $41 \%$ of disabling headaches and $12 \%$ of neurological progressive deficits, with stroke-like symptoms and even transitory ischemic attacks ${ }^{3}$.

Despite the absence of seizures, our patient is a young female with relevant complaints of headache, left parestesis and intermittent paresis, totally related to CPA physiopathology, concerning "nidus" hyperperfusion associat- 
ed with hemispheric hypoperfusion, characteristic signals of hemodynamic steal phenomenon.

The main differences between CPA and typical AVMs are the numerous non dominant feeders including transdural supply to lobar or even hemispheric "nidus", moderately enlarged draining veins and normal brain tissue intermingled between vascular spaces. Increased blood volume within the nidus and delayed blood transit time are predictive of the ectatic capillary net formation ${ }^{3}$.

In fact, there is a progressive vicious cascade in which arterial supply associated with venous ectasia creates an environment of local increased blood volume and perinidal areas of severely hypoperfusion triggering an uncontrolled progressive angiogenic response to this normal brain with abnormal blood demand.

Treatment of AVMs, based on the Spetzler-Martin's classification, may be done by surgical resection, embolization and radiosurger $\mathrm{y}^{7-10}$. In CPA however, the normal brain tissue between vascular spaces carry the risk of permanent neurological deficit. Headache may be dramatically alleviated by limited arterial embolization in noneloquent areas. As the major pathomechanism of this disease is ischemia owing to incompetent angiogenesis; seizures, headaches and steal phenomenon, similar to MoyaMoya-like diseases, may be treated with calvarial burrholes that increases cortical blood supply by recruiting additional dural blood supply ${ }^{3}$.

The main propose of this case report is indeed the knowledge of the CPA, as a differential diagnosis of AVM syndromes, once an AVM diagnose mistake implicate in potentially lethal results if surgery or embolization would be performed.

Vast bibliographical revision makes evidence of the extreme rare incidence of cerebral proliferative angiopathy which enhances the paramount importance of the case.

\section{REFERENCES}

1. Yasargil MG AVM of the brain, history, embryology, pathological considerations, hemodynamics, diagnostics, studies, microsurgical anatomy. In: Microneurosurgery IIIA. New York: Thieme, 1987:3-21.

2. Schreiber SJ, Doepp F, Bender A, Schmierer K, Valdueza J M. Difuse cerebral angiomatosis. Neurology 2003;60:1218-1219.

3. Lasjaunias PL, Landrieu P, Rodesch G, et al. Cerebral proliferative angiopathy: clinical and angiographic description of an entity different from cerebral AVMs. Stroke 2008;39:878-885.

4. Shovlin CL, Guttmacher AE, Buscarini E, et al. Diagnostic criteria for hereditary hemorrhagic telangiectasy (Rendu-Osler-Weber syndrome). Am J Med Genet 2000; 91:66-67.

5. Sujansky E, Conradi S. Sturge Weber syndrome: age of onset of seizures and glaucoma and prognosis for affected children. J Child Neurol 1995;10:49-58.

6. Ponce FA, Han PP, Spetzler RF, et al. Associated arteriovenous malformation of the orbit and brain: a case of Wyburn-Mason syndrome without retinal involvement. Case report. J Neurosurg 2001;95:346-349.

7. Chaddad-Neto FEA, Fernandes J A, Tadeu S, et al. Microsurgical approach of arteriovenous malformations in the central lobule. Arq Neuropsiquiatr 2008; 66:872-875.

8. Hashimoto N, Nozaki K, Takagi Y, Kikuta K, Mikuni N. Surgery of cerebral arteriovenous malformations. Neurosurgery 2007;61:375-389.

9. Spetzler RF, Martin NA. A proposed grading system for arteriovenous malformations. J Neurosurg 1986;65:476-483.

10. Takagi Y, kikuta K, Nozaki K, Fujimoto M, Hayashi J, Hashimoto N. Neuronal expression of Fas-associated death domain protein and caspase 8 in the perinidal parenchyma of cerebral arteriovenous malformations. J Neurosurg 2007; 106:275-282. 